\title{
Interaction corrections to transport due to quasibound states in open quantum dots
}

\author{
K. M. Indlekofer, ${ }^{\text {a) }}$ J. P. Bird, R. Akis, D. K. Ferry, and S. M. Goodnick \\ Department of Electrical Engineering and Center for Solid State Electronics Research, \\ Arizona State University, Tempe, Arizona 85287-5706
}

(Received 3 July 2002; accepted 20 September 2002)

\begin{abstract}
We formulate a many-body model of transport in open quantum dots, which is based upon the idea of an enhanced electron-electron interaction in the vicinity of a quasibound state. Our studies suggest that the effect of including this peaked interaction is to increase the amplitude of the conductance fluctuations, beyond the value expected from a single-particle treatment. While the role of interactions in transport through open dots has attracted little theoretical attention, our results demonstrate the presence of interaction-induced corrections to the transport in these structures.

(C) 2002 American Institute of Physics. [DOI: 10.1063/1.1521584]
\end{abstract}

Recent experimental and theoretical studies of open quantum dots have revealed a picture in which the transport through these structures is dominated by the contribution from a small number of eigenstates of the corresponding closed structure, which remain resolved when the dot is coupled to external reservoirs by means of one-dimensional leads. ${ }^{1-5}$ The robust eigenstates are found to be scarred by the remnants of isolated periodic orbits (Fig. 1, lower inset), which buildup localized regions of high probability density within the center of the dot. Measurable transport results associated with the scars have been demonstrated in experiment, ${ }^{3}$ and, in a recent semiclassical analysis, this behavior was shown to be consistent with a process in which electrons dynamically tunnel through forbidden regions of the classical phase space, to access isolated periodic orbits. ${ }^{4}$ While these single-particle based theoretical approaches appear to provide a good description of transport in the open dots, we note that the dynamical tunneling implies the existence of certain resonant energies, at which electrons are quasibound in the dot and can only escape on time scales much longer than the direct transit time. ${ }^{5}$ For this reason, it is of interest to explore the existence of many-body corrections to the transport, which arise from an enhancement of the electron interaction in the presence of the resonant-trapping effect. Indeed, in a series of recent experimental reports, we have discussed the presence of features in the electrical properties of open dots which are believed to be associated with such many-body interactions. ${ }^{6-8}$

In this letter, we formulate a many-body model of transport in open quantum dots, which is based upon the idea of an enhanced electron-electron interaction in the vicinity of a quasibound state. Our studies suggest that the effect of including this peaked interaction is to increase the amplitude of the conductance fluctuations, beyond the value expected from a single-particle treatment. While the role of interactions in transport through open dots has thus far attracted little theoretical attention, our results demonstrate the presence of interaction-induced corrections to the transport in these structures. We also consider the relevance of our model

${ }^{\text {a)} E l e c t r o n i c ~ m a i l: ~ M . I n d l e k o f e r @ f z-j u e l i c h . d e ~}$ to the earlier-mentioned experimental studies of open quantum dots, which have demonstrated unexpected results at low temperatures, where interaction effects are thought to be pronounced. ${ }^{6-8}$

The key experimental observations to this study are summarized in Fig. 1, which shows the temperature dependence of the resistance of a split-gate quantum-dot array, for two different gate-bias conditions. Although not shown here, at low temperatures the resistance of these dots exhibits reproducible fluctuations, which may be generated by varying ei-

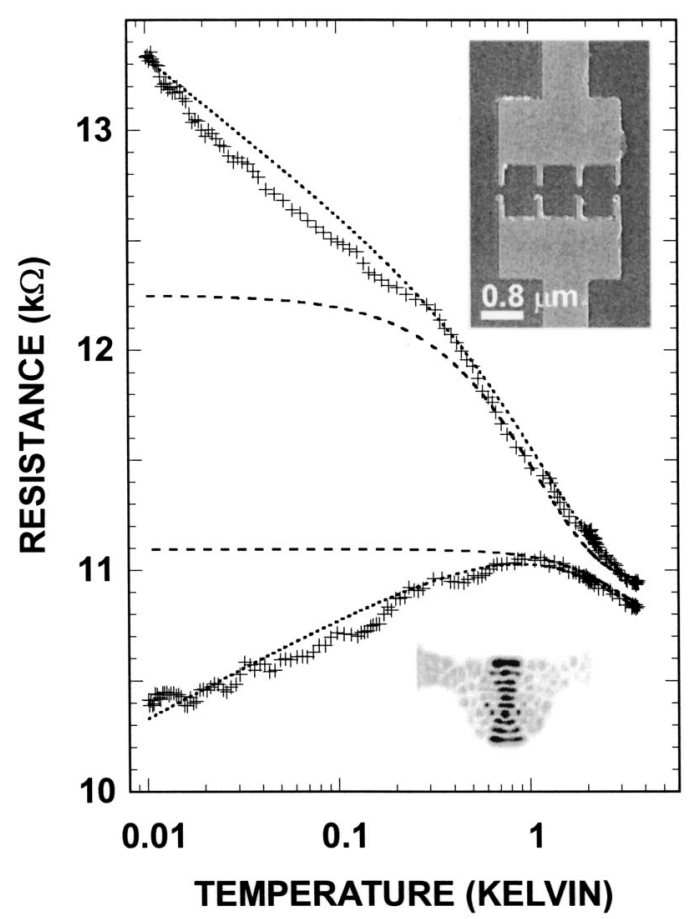

FIG. 1. Measured variation of resistance with temperature in a split gate quantum-dot array. The-dotted line represents a fit to the form of Eq. (1) with parameters $R_{0}=11.3(10.1) \mathrm{k} \Omega, R_{1}=0.65(1.15) \mathrm{k} \Omega, R_{2}=-0.3(+0.2)$ $\mathrm{k} \Omega, T_{0}=1.1(4.0) \mathrm{K}$, and $p=1.3$ (1.1) for the upper (lower) curve. Data shown is from device $B$ of Ref. 7. The dashed lines are fits without a low-temperature logarithmic term $\left[R_{0}=11.0(10.8) \mathrm{k} \Omega, R_{1}=1.3(0.3) \mathrm{k} \Omega\right.$, $T_{0}=1.1(3.0) \mathrm{K}$, and $\left.p=1.3(2.0)\right]$. Upper inset shows a scanning electron microscopy micrograph of device $A$ from Ref. 7. Lower inset is a calculated scar for the device studied in Ref. 3. 
ther the gate voltage or the external magnetic field. ${ }^{3,4,6-8}$ The data shown in Fig. 1 are quite representative $e^{7,8}$ and were obtained by setting the gate voltage to correspond to a local maximum (upper curve) and minimum in the lowtemperature resistance versus gate voltage curve. ${ }^{6-8}$ The dotted line through each data set represents a fit of the resistance to the following form:

$$
R(T)=R_{0}+R_{1} \exp \left[\left(\frac{T_{0}}{T}\right)^{p}\right]+R_{2} \ln T,
$$

where $T$ is the temperature, and $R_{0}, R_{1}, R_{2}, T_{0}$, and $p$ are fit parameters. ${ }^{8}$ In our experimental investigations of these variations, we have suggested that the exponential term in Eq. (1) arises from the resolution of the discrete dot levels with decreasing temperature, ${ }^{8}$ while the $\ln T$ term is thought to be a signature of many-body interactions, since it is not reproduced in single-particle treatments.

The theoretical model that we explore is based on the idea of the Anderson system, ${ }^{9-15}$ where a single impurity level with an on-site electron-electron interaction is coupled to a one-dimensional lead. In our case, the single impurity is interpreted as the quantum dot, which is coupled to two quantum-point-contact leads. To obtain a many-body model for the interacting open system, however, we start with the single-particle eigenbasis of the integral dot/lead system with one lateral mode. The propagating one-dimensional eigenstates of this system are identified by their wave number $(k)$ in the point contacts (where the sign of $k$ defines the source). At certain energies, the transmission of this mode can exhibit a Fano-type resonance, reflecting the presence of a quasibound state which exhibits a resonantly enhanced charge density that is localized within the dot. ${ }^{5}$ In this situation, the resulting effective-interaction matrix elements will also exhibit a resonance and the model Hamiltonian which should describe this interaction for one mode propagating through a single open dot may be written as

$$
\begin{aligned}
\hat{H}= & -\beta\left[\sum_{k, \sigma}\left(\epsilon_{k \sigma}-\mu_{k}\right) c_{k \sigma}^{+} c_{k \sigma}\right. \\
& \left.+\frac{1}{2} \sum_{(k \sigma) \neq\left(k^{\prime} \sigma^{\prime}\right)} V_{(k \sigma)\left(k^{\prime} \sigma^{\prime}\right)} c_{k \sigma}^{+} c_{k \sigma} c_{k^{\prime} \sigma^{\prime}}^{+} c_{k^{\prime} \sigma^{\prime}}\right],
\end{aligned}
$$

where $\beta=1 / k_{B} T, \mu_{k}$ is the electrochemical potential associated with the mode with wave number $k, \sigma$ denotes the usual spin index, $c_{k \sigma}^{+} / c_{k \sigma}$ are the usual creation/annihilation operators, and $\epsilon_{k \sigma}$ denotes the single-particle dispersion relation. The applied voltage between the injecting electron reservoirs corresponds to the difference in chemical potentials, $\mu_{k>0}$ $-\mu_{k<0}$. For the effective interaction matrix $V$, we employ the following peaked expression in the vicinity of a resonance $\left(E_{0}\right)$ :

$$
\begin{aligned}
V_{(k \sigma)\left(k^{\prime} \sigma^{\prime}\right)} & \left(U-\delta_{\sigma \sigma^{\prime}} U^{\mathrm{ex}}\right) \\
& \times \frac{\Gamma^{2}}{\left(\epsilon_{k}-E_{0}\right)^{2}+\Gamma^{2}} \frac{\Gamma^{2}}{\left(\epsilon_{k^{\prime}}-E_{0}\right)^{2}+\Gamma^{2}} .
\end{aligned}
$$

Here, $U$ and $U^{\text {ex }}$ are the Hartree and exchange terms, respectively, and $2 \Gamma$ denotes the total width of the interaction resonance, corresponding to the lifetime of the resonantly trapped charge. Note here that screening within any particuDownloaded 15 Dec 2006 to 134.94.122.39. Redistribution subject

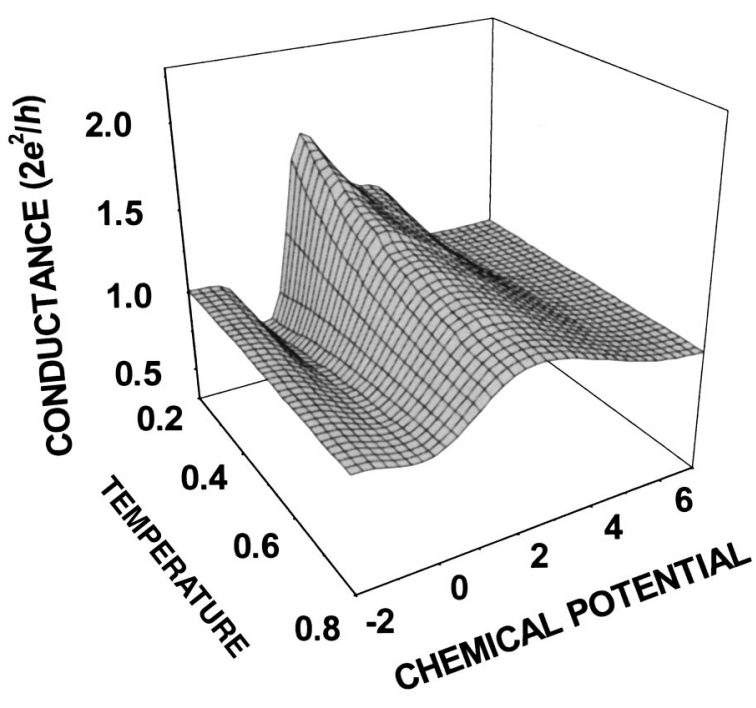

FIG. 2. Normalized conductance as a function of the chemical potential $\mu$ (relative to $E_{0}$ ) and the temperature $T$ for $\Gamma=0.4, U=2.4, U^{\mathrm{ex}}=0.6$ (with a total number of $12 k$ states).

lar mode is included explicitly in this many-body Hamiltonian, whereas screening effects arising from propagating background modes, and the environment, are assumed to be contained within the effective interaction matrix $V$.

To calculate transport properties from this model, we consider the diagonal nonequilibrium (due the nonconstant chemical potential) many-body density-matrix $\rho$ $=Z^{-1} \exp (\hat{H})$, with the partition function $Z$. For numerical reasons, the continuum of $k$ states is discretized in our simulations, and we further employ a mean-field approach for the calculation of occupation numbers for state numbers larger than 16 (the discretization in $k$ space must be chosen such that the resulting energy spacing is small compared to $\left.k_{B} T\right) .{ }^{16}$ The current flowing through the system (and, hence, the conductance) can then be expressed in terms of the single-particle transmission probability (with its Fano-type resonance) and the occupation numbers. ${ }^{16}$ Figure 2 shows the typical dependence of the conductance correction due to the interaction (assuming unity transmission) with varying chemical potential and temperature (all quantities in arbitrary units). The conductance valley can be understood as a depletion of current-carrying $k$ states at energies below the interaction peak. After the interaction has been overcome by the chemical potential, an enhanced density of states contributes to the current, and a conductance peak therefore results. A systematic analysis ${ }^{16}$ leads us to define a characteristic energy scale $k_{B} T^{*}=\alpha\left(\Gamma V_{\text {tot }}^{\text {state }}\right)^{0.5}$, with fitting parameters $\alpha^{G} \approx 0.55$ and $\alpha^{R} \approx 0.31$ for the conductance and resistance maxima, respectively. $V_{\text {tot }}^{\text {state }}$ denotes the normalized interaction energy per state, and we note that the temperature $T^{*}$ has a form reminiscent of an effective Kondo temperature. ${ }^{9-16}$ In the high-temperature regime $\left(T \gg T^{*}\right)$, we find a modified Boltzmann law for the scaling of the corrections to the local conductance and resistance peaks

$$
G_{\text {peak }}^{\text {corr }} \propto \exp \left[\left(\frac{T_{G}^{*}}{T}\right)^{2}\right], \quad R_{\text {peak }}^{\text {corr }} \propto \exp \left[\left(\frac{T_{R}^{*}}{T}\right)^{2}\right] .
$$




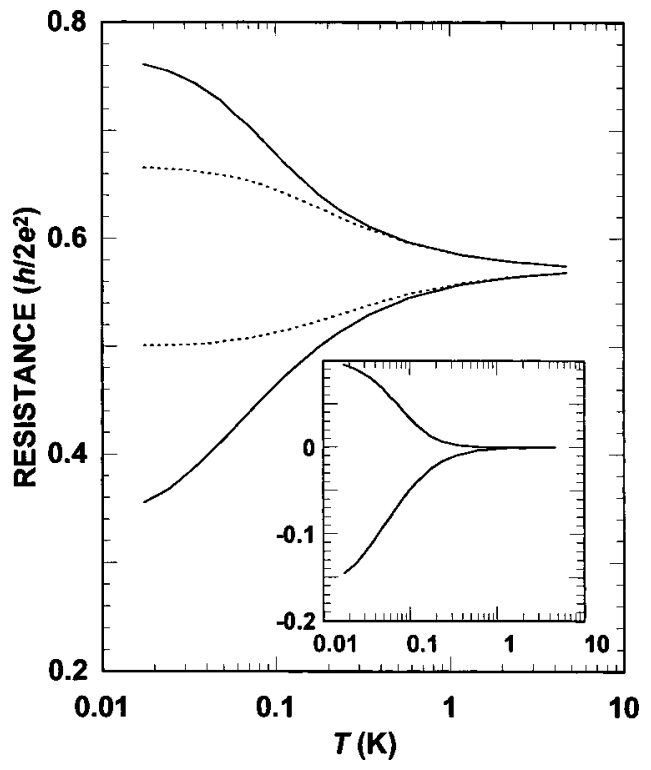

FIG. 3. Computed temperature dependence of the resistance peak (upper curve) and valley associated with a specific peaked interaction. The calculation includes $128 k$ states and the solid lines correspond to the case where interactions are included, while the dotted lines are for no interaction. The inset shows the corresponding interaction corrections determined from these data (i.e., the difference between the interacting and noninteracting cases).

conductance correction approximately proportional to $\ln T$ is found, which is surprisingly similar to the behavior found in experiment (Fig. 1). ${ }^{7}$

To compare the predictions of the peaked-interaction model with experiment, we show the results of a more realistic simulation in Fig. 3. This now includes the Fano-type resonance $^{5}$ in the single-particle transmission probability. Here we have taken $\Gamma=0.02 \mathrm{meV}, U=0.125 \mathrm{meV}$, and $U^{\mathrm{ex}}$ $=0.5 U$, a parameter choice which yields $V_{\text {tot }}^{\text {state }} \approx 0.010 \mathrm{meV}$, $T_{G}^{*} \approx 95 \mathrm{mK}$ and $T_{R}^{*} \approx 53 \mathrm{mK}$. We have also assumed a Fanolike transmission probability with a peak ${ }^{5}$ at $E_{p}=\Gamma$, a zero at $E_{0}=0$, a total amplitude prefactor of 0.5 , and a background transmission of 0.5 . Furthermore, we have assumed that we have one nonresonant background mode (with a conductance of $\left.2 e^{2} / h\right)$. In Fig. 3, the dotted curves show the variation of the peak- and valley-resistance values as a function of the temperature, which were computed without the inclusion of any interaction terms (that is, this figure shows the temperature behavior due to the single-particle Fano-resonance of the mode). The solid lines in the figure correspond to the situation where the peaked interaction term has been included in the simulation. Clearly, the interaction yields an increased fluctuation amplitude, compared to the singleparticle behavior. In the inset to Fig. 3, the difference between the interacting and noninteracting cases is plotted (for the peak and the valley as two separate curves), and, in the limited temperature range close to $T^{*}$, one can see a log-like correction due to the peaked interaction, as a first-order correction beyond the single-particle case. The qualitative simi- larity with the experimental results of Fig. 1 is suggestive, and a further interesting observation is that the interaction corrections to the resistance valley (i.e., conduction peak) onset at higher temperatures than for the resistance peak, which can also be seen in the experiment. We do not suggest, however, that the agreement between experiment and theory is complete. In fact, while we have investigated the behavior for a single propagating mode (for ease of implementation), ${ }^{16}$ in experiment one typically deals with dot-array structures in which many propagating modes, and temperature-dependent dephasing effects, are present. ${ }^{6-8}$ To conclusively associate the features observed in experiment with many-body effects, it will therefore be necessary to extend this model to account for these differences. In spite of this, however, we are encouraged by the fact that our rather simple model does indeed yield interaction-induced corrections to the conductance of open dots.

In conclusion, we have formulated a many-body model of transport in open quantum dots, which is based upon the idea of an enhanced electron-electron interaction in the vicinity of a quasibound state. Our studies suggest that the effect of including this peaked interaction is to increase the amplitude of the conductance fluctuations, beyond the value expected from a single-particle treatment. While the role of interactions in transport through open dots has thus far attracted little theoretical attention, our results therefore demonstrate the presence of interaction-induced corrections to the transport in these structures.

This work was supported by the Office of Naval Research, the Department of Energy (J.P.B.), and the Alexander von Humboldt Foundation (K.M.I.).

${ }^{1}$ R. Akis, D. K. Ferry, and J. P. Bird, Phys. Rev. B 54, 17705 (1996).

${ }^{2}$ R. Akis, D. K. Ferry, and J. P. Bird, Phys. Rev. Lett. 79, 123 (1997).

${ }^{3}$ J. P. Bird, R. Akis, D. K. Ferry, D. Vasileska, J. Cooper, Y. Aoyagi, and T. Sugano, Phys. Rev. Lett. 82, 4691 (1999).

${ }^{4}$ A. P. S. de Moura, Y.-C. Lai, R. Akis, J. P. Bird, and D. K. Ferry, Phys. Rev. Lett. 88, 236804 (2002).

${ }^{5}$ R. Akis, J. P. Bird, and D. K. Ferry, Appl. Phys. Lett. 81, 129 (2002).

${ }^{6}$ L.-H. Lin, N. Aoki, K. Nakao, A. Andresen, C. Prasad, F. Ge, J. P. Bird, D. K. Ferry, Y. Ochiai, K. Ishibashi, Y. Aoyagi, and T. Sugano, Phys. Rev. B 60, R16299 (1999).

${ }^{7}$ A. Shailos, J. P. Bird, C. Prasad, M. Elhassan, L. Shifren, D. K. Ferry, L.-H. Lin, N. Aoki, Y. Ochiai, K Ishibashi, and Y. Aoyagi, Phys. Rev. B 63, 241302 (2001).

${ }^{8}$ A. Shailos, C. Prasad, M. Elhassan, R. Akis, D. K. Ferry, J. P. Bird, N. Aoki, L.-H. Lin, Y. Ochiai, K. Ishibashi, and Y. Aoyagi, Phys. Rev. B 64, 193302 (2001).

${ }^{9}$ P. W. Anderson, Phys. Rev. 124, 41 (1961).

${ }^{10}$ T. K. Ng and P. A. Lee, Phys. Rev. Lett. 61, 1768 (1988).

${ }^{11}$ Y. Meir, N. S. Wingreen, and P. A. Lee, Phys. Rev. Lett. 66, 3048 (1991).

${ }^{12}$ Y. Meir, N. S. Wingreen, and P. A. Lee, Phys. Rev. Lett. 70, 2601 (1993).

${ }^{13}$ N. S. Wingreen and Y. Meir, Phys. Rev. B 49, 11040 (1994).

${ }^{14}$ D. Goldhaber-Gordon, J. Göres, M. A. Kastner, H. Shtrikman, D. Mahalu, and U. Meirav, Phys. Rev. Lett. 81, 5225 (1998).

${ }^{15}$ W. G. Van der Wiel, S. De Franceschi, T. Fujisawa, J. M. Elzerman, S. Tarucha, and L. P. Kouwenhoven, Science 289, 2105 (2000).

${ }^{16}$ K. M. Indlekofer, R. Akis, J. P. Bird, D. K. Ferry, and S. M. Goodnick (unpublished). 